\title{
Effects of Temperature and Packaging on the Growth Kinetics of Clostridium perfringens in Ready-to-eat Jokbal (Pig's Trotters)
}

\author{
Hee-Jin Park, Yu-Jin Na, Joon-II Cho', Soon-Ho Lee ${ }^{2}$, and Ki-Sun Yoon* \\ Department of Food and Nutrition, Kyung Hee University, Seoul 130-701, Korea \\ ${ }^{1}$ Food Microbiology Division, Ministry of Food and Drug Safety, Cheongwon 363-700, Korea \\ ${ }^{2}$ Foodborne Diseases Prevention and Surveillance Division, Ministry of Food and Drug Safety, Cheongwon 363-700, Korea
}

\begin{abstract}
Ready-to-eat (RTE) Jokbal (Pig's trotter), which consists of pig's feet cooked in soy sauce and various spices, is a very popular and widely sold in Korean retail markets. Commercially, the anaerobically packed Jokbal have also become a popular RTE food in several convenience stores. This study evaluates the effects of storage temperature and packaging methods for the growth of $C$. perfringens in Jokbal. Growth kinetic parameters of $C$. perfringens in aerobically and anaerobically packed Jokbals are determined at each temperature by the modified Gompertz equation. The lag time, specific growth rate, and maximum population density of $C$. perfringens are being analyzed as a function of temperature and packaging method. The minimum growth temperature of C. perfringens in aerobically and anaerobically packed $\mathrm{Jokbal}$ is $24^{\circ} \mathrm{C}$ and $18^{\circ} \mathrm{C}$, respectively. The $C$. perfringens in Jokbal did not grow under conditions of over $50^{\circ} \mathrm{C}$ regardless of the packaging methods, indicating that the holding temperature of Jokbal in markets must be maintained at above $50^{\circ} \mathrm{C}$ or below $18^{\circ} \mathrm{C}$. Growth of C. perfringens in anaerobically packed Jokbal is faster than in aerobically packed Jokbal when stored under the same conditions. This indicates that there are a higher risks associated with $C$. perfringens for anaerobically packed meat products.
\end{abstract}

Key words: Clostridiun perfringens, RTE Jokbal, growth model, temperature, packaging method

\section{Introduction}

Recently foodborne outbreaks associated with Clostridium perfringens have been steadily increasing. During the past three years in Korea, there have been 25 outbreaks and 792 cases reported as $C$. perfringens associated with foodborne illness (MFDS, 2013). Most of the cases of $C$. perfringens associated with food poisoning have occurred in institutions and food service establishments where large amounts of food are cooked in advance prior to serving. Thus, food poisoning outbreak due to C. perfringens was reported with the highest number of case per outbreak.

C. perfringens is mainly found in protein-rich foods with $75 \%$ of foodborne outbreaks being traced to meat and meat products (Brynestad and Granum, 2002; Johnson and Gerding, 1997). Vehicles for C. perfringens outbreaks include roast beef, turkey, meat-containing Mexican food,

*Corresponding author: Ki-Sun Yoon, Department of Food and Nutrition, Research Institute of Human Ecology, Kyung Hee University, Seoul 130-701, Korea. Tel: 82-2-961-0264, Fax: 822-961-0261, E-mail: ksyoon@khu.ac.kr stew, salmon, lasagna, reindeer, and anaerobically packed pork (Bryan, 1988; Hatheway, 1990).

Meat products that are thermally processed and do not require further heating by consumers are classified as RTE foods. Golden et al. (2009) conducted risk assessment for C. perfringens in Ready-to-eat (RTE) and in partially cooked meat products. The major risk factor (91\%) for illnesses associated with $C$. perfringens is growth during retail and consumer storage. Another risk factor (7.6\%) for illnesses related to C. perfringens is that of improper initial heating. C. perfringens has the ability to form heat-resistant endospores which allows it to survive in improperly cooked food. Furthermore these spores grow quickly in protein-rich foods, which allows $C$. perfringens to rapidly replicate to the level necessary to initiate infection even with low contamination (Labbe and Harmon, 1992). Thus it is important to control C. perfringens growth at retail markets as well as in food service establishments in order to reduce outbreaks associated with $C$. perfringens.

RTE Jokbal consists of pig's trotter cooked with soy sauce and spices, which is a very popular and widely sold in Korean retail markets. Recently a food consumption 
survey study was conducted on 50 potentially hazardous foods (PHF) in Korea (Park et al., 2013). About 36\% of the respondents ate Jokbal more than once per month. Amount consumed in a single sitting was $170.6 \mathrm{~g}$, which is $170.6 \%$ of the recommended serving. $76.7 \%$ of the respondents purchased Jokbal in a restaurant, which was followed by traditional markets $(9.7 \%)$, supermarkets $(6.8 \%)$, and retail markets (5.5\%). $80.1 \%$ of the respondents bought the Jokbal stored at room temperature.

Predictive food microbiology can be defined as the use of mathematical expressions to describe microbial behavior in food production. This is an efficient way to control the microbiological safety of meat products. Growth or survival predictive models have also been a very useful tool in microbial risk assessment and are thus an important tool used by the Hazard Analysis Critical Control Point (HACCP) program. Previous studies have developed predictive models for $C$. perfringens in various meats (Juneja et al., 2008, 2010; Le Marc et al., 2008), but these models are not appropriate for assessing risk in the popular and widely sold RTE Jokbal.

In the present study, the effects of storage temperature and packaging method (aerobic and anaerobic) on the growth kinetics of $C$. perfringens in Jokbal was investigated and a predictive growth model of $C$. perfringens was also developed as a function of temperature and packaging method.

\section{Material and Methods}

\section{Bacterial strains and sample preparation}

An $\alpha$-toxin producing strain of $C$. perfringens ATCC 13124 was purchased from the Korean Culture Center for Microorganism (Korea). For each experiment, stock cultures of $C$. perfringens were thawed at room temperature. Fifty microliter of thawed stock was added into a sterile polypropylene coning tube (SPL, Korea) containing $5 \mathrm{~mL}$ of sterile Reinforced Clostridium medium (RCM) broth. It was placed in a microbial anaerobic jar (Merck, Germany), which was then injected with modified gas $\left(\mathrm{H}_{2}\right.$ $5 \%, \mathrm{CO}_{2} 10 \%$, and $\mathrm{N}_{2} 85 \%$ ) by a gas exchange device (Don Whitley Scientific, UK) and incubated at $37^{\circ} \mathrm{C}$ for 24 h. RTE Jokbals were purchased from a retail market in Seoul, Korea. The Jokbals were heated in a microwave for $1 \mathrm{~min}$ and $10 \mathrm{~g}$ of RTE Jokbals were aseptically weighed and placed in a sterile petri dish. Each thin slice of RTE Jokbal was then uniformly surface inoculated with $100 \mu \mathrm{L}$ of the diluted starter culture of $C$. perfringens using a sterile pipette to give a target population of approximately 2.5-3.5 Log CFU/g. Samples were packed aerobically or anaerobically (Fresh pack FM-03, Eiffel, Korea) and stored at $18,20,24,28,36,44$, and $50^{\circ} \mathrm{C}$.

\section{Microbial analysis}

The samples were homogenized with $90 \mathrm{~mL}$ of sterile $0.1 \%$ peptone water in a stomacher lab blender (Bag Mixer 400, Interscience, France) for $2 \mathrm{~min}$. Serial dilutions were prepared with sterile peptone water and $100 \mu \mathrm{L}$ aliquots were taken and plated onto a thin layer $(15 \mathrm{~mL})$ of tryptose-sulfite-cycloserine (TSC; Oxoid, UK) agar without egg yolk. A modified plating technique was used (dual-layer pour plating) for pour plating. TSC agar plates were overlaid with an additional $5 \mathrm{~mL}$ of TSC agar. All plates were incubated for $24 \mathrm{~h}$ at $37^{\circ} \mathrm{C}$ in a microbial anaerobic jar, which was injected with modified gas $\left(\mathrm{H}_{2}\right.$ $5 \%, \mathrm{CO}_{2} 10 \%$, and $\mathrm{N}_{2} 85 \%$ ) by a gas exchange device. Typical black colonies were counted as Log CFU/g (Colony Counter SCAN 1200, Interscience, France).

\section{Primary model development}

The growth kinetic parameters including lag time (LT) and specific growth rate (SGR) in the primary model at each temperature were determined by the modified Gompertz equation (Gibson et al., 1987) using GraphPad Prism V4.0 (GraphPad Sofrware, USA).

$$
\begin{aligned}
& \text { Gompertz model : } \\
& \mathrm{Y}=\mathrm{N}_{0}+\mathrm{C} \times \exp (-\exp ((2.718 \times \mathrm{SGR} / \mathrm{C}) \times(\mathrm{T}-\mathrm{t})+1))
\end{aligned}
$$

where $\mathrm{Y}$ is the viable cell count $(\mathrm{Log} \mathrm{CFU} / \mathrm{g}$ ) at time $\mathrm{t}$ (h), $\mathrm{N}_{0}$ is the initial $\log$ number of cells, $\mathrm{C}$ is the difference between the initial and final cell numbers, SGR is the maximum specific growth rate ( $\log \mathrm{CFU} / \mathrm{h}), \mathrm{T}$ is the lag time before growth, and $t$ is sampling time. Each experiment was replicated twice. The goodness-of-fit of the data was evaluated based on the coefficient of determination $\left(\mathrm{R}^{2}\right)$, which was calculated by GraphPad Prism.

\section{Secondary model development}

LT, SGR, and MPD values graphed as a function of temperature and then fitted to the Davey, Square-root, and second order polynomial equations, respectively. The Davey model used was as follows (Daughtry et al., 1997):

$$
\mathrm{Y}=\mathrm{a}+(\mathrm{b} / \mathrm{T})+\left(\mathrm{c} / \mathrm{T}^{2}\right)
$$

where $\mathrm{Y}$ is LT (day), a, b, and c are regression coefficients without biological meaning, and $\mathrm{T}$ is the tempera- 
ture.

The square-root model used was as follows (Ratkowsky et al., 1982):

$$
\sqrt{\mathrm{Y}}=\mathrm{b}\left(\mathrm{T}-\mathrm{T}_{\min }\right)
$$

where $\mathrm{Y}$ is $\mathrm{SGR}$ (Log CFU/day), b is a regression coefficient, $\mathrm{T}_{\min }$ is the cardinal minimum growth temperature.

The second order polynomial model used was as follows (McMeekin et al., 1993):

$$
\mathrm{Y}=\mathrm{a}+\mathrm{bT}+\mathrm{cT} \mathrm{T}^{2}
$$

where $\mathrm{Y}$ is the maximum population density ( $\log \mathrm{CFU}$ ), $\mathrm{a}, \mathrm{b}$, and $\mathrm{c}$ are regression coefficients without biological meaning and $\mathrm{T}$ is the temperature.

\section{Model performance}

The performance of the models was quantified using the ratio method described by Ross (1996).

$$
\begin{aligned}
& B_{f} \text { for } \mathrm{LT}=10^{\log (\text { predicted } / \text { observed }) / n} \\
& A_{f} \text { for } \mathrm{LT}=10^{[\log (\text { predicted } / \text { observed }) / n]}
\end{aligned}
$$

$$
\begin{aligned}
& B_{f} \text { for } \mathrm{SGR} \text { or MPD }=10^{\log (\text { observed } / \text { predicted }) / n} \\
& A_{f} \text { for } \mathrm{SGR} \text { or MPD }=10^{[\log (\text { observed/predicted }) / n]}
\end{aligned}
$$

where $\mathrm{n}$ is the number of prediction cases used in the calculation. $B_{f}$ values consider whether prediction error is more in the fail safe direction or not, while $A_{f}$ values do not consider the direction of prediction error. Thus different ratios were used for LT, SGR, and MPD, so that $B_{f}$ less than 1 would represent fail-safe predictions, and $B_{f}$ above 1 would represent fail-dangerous predictions (Abou-Zeid et al., 2009)

In addition, relative error (RE) of individual prediction cases was calculated using the following equation (Delignette-Muller et al., 1995).

$$
\mathrm{RE} \text { for } \mathrm{LT}=[(\text { predicted-observed }) / \text { predicted }]
$$

\begin{tabular}{|c|c|c|c|c|}
\hline Temperature $\left({ }^{\circ} \mathrm{C}\right)$ & Parameter & Anaerobic & Aerobic & $\overline{t \text { t-value }}$ \\
\hline \multirow{3}{*}{18} & ${ }^{1)} \mathrm{LT}$ & 20.13 & - & - \\
\hline & ${ }^{2)} \mathrm{SGR}$ & 0.19 & - & - \\
\hline & ${ }^{3)}$ MPD & 8.26 & - & - \\
\hline \multirow{3}{*}{20} & LT & 13.26 & - & - \\
\hline & SGR & 0.08 & - & - \\
\hline & MPD & 8.39 & - & - \\
\hline \multirow{3}{*}{24} & LT & - & 12.3 & - \\
\hline & SGR & - & 0.06 & - \\
\hline & MPD & - & 7.74 & - \\
\hline \multirow{3}{*}{28} & LT & 5.46 & 8.53 & -1.53 \\
\hline & SGR & 0.56 & 0.24 & 1.70 \\
\hline & MPD & 8.61 & 7.94 & $15.39^{*}$ \\
\hline \multirow{3}{*}{36} & LT & 3.44 & 3.10 & 4.72 \\
\hline & SGR & 0.97 & 0.64 & 1.22 \\
\hline & MPD & 8.76 & 8.45 & 3.44 \\
\hline \multirow{3}{*}{44} & LT & 1.91 & 2.16 & -0.90 \\
\hline & SGR & 1.57 & 1.24 & 1.92 \\
\hline & MPD & 8.56 & 8.27 & 4.72 \\
\hline \multirow{3}{*}{50} & LT & 1.29 & 2.35 & -2.29 \\
\hline & SGR & 2.21 & 2.34 & -0.28 \\
\hline & MPD & 8.16 & 7.76 & $9.33^{*}$ \\
\hline
\end{tabular}

$$
\begin{aligned}
& \mathrm{RE} \text { for SGR or MPD }= \\
& {[(\text { observed-predicted }) / \text { predicted }]}
\end{aligned}
$$

The median relative error (MRE) and the mean absolute relative error (MARE) were also used to measure the prediction bias and accuracy of the model, respectively.

Table 1. Growth kinetics of $C$. perfringens in anaerobically and aerobically packed RTE Jokbals as a function of temperature

1) LT: Lag time (h)

${ }^{2}$ SGR: Specific growth rate (Log CFU/h)

${ }^{3)} \mathrm{MPD}$ : Maximum population density (Log CFU)

${ }^{*} p<0.05$ 


\section{Statistics analysis}

Experiments were replicated as previously indicated. Data were analyzed with SAS software, version 9.3 (SAS Institute, Inc., USA) with log-transformed data. Analyses of variance were performed using ANOVA and a t-test was also conducted for comparison between samples. Significant $(p<0.05)$ differences between the means of duplicate measurements in each independent trial were determined with Duncan's multiple range test.

\section{Results and Discussion}

Effect of storage temperature and packaging on the growth kinetics of $C$. perfringens

Effect of storage temperature and packaging method on the growth kinetics of $C$. perfringens, including lag time (LT), specific growth rate (SGR), and maximum population density (MPD) were studied in RTE Jokbal (Table 1). In addition, primary growth models for $C$. perfringens in anaerobically and aerobically packed Jokbals were developed at each storage temperature and are shown in Fig. 1. The growth curves in RTE Jokbal fitted well to a modified Gompertz equation $\left(\mathrm{R}^{2}=0.9689\right.$ to 0.9958$)$. Overall, the growth of $C$. perfringens in anaerobically packed Jokbal was more rapid than that in aerobically packed Jokbal. The minimum growth temperatures were also different depending on packaging method. $C$. perfringens in anaerobically packed Jokbal was grown at the temperature range of 18 to $50^{\circ} \mathrm{C}$, while the growth of $C$. perfringens in aerobically packed Jokbal was not observed at the temperature lower than $24^{\circ} \mathrm{C}$. Overall, LT values of $C$. perfringens in aerobically packed Jokbal were longer than those in anaerobically packed Jokbal. The LT of C. perfringens in anaerobically packed Jokbal stored at $18^{\circ} \mathrm{C}$ was approximately 15 times longer than that at $50^{\circ} \mathrm{C}$. SGR value of $C$. perfringens in anaerobically packed RTE Jokbal stored at $18^{\circ} \mathrm{C}$ and $36^{\circ} \mathrm{C}$ was 11.9 and 2.3 times slower, respectively, compared to that at $50^{\circ} \mathrm{C}$. Also the SGR of (a)

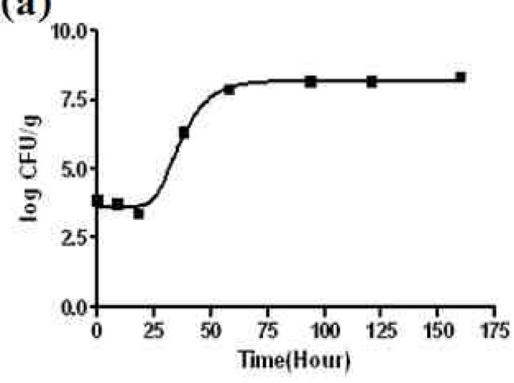

(c)

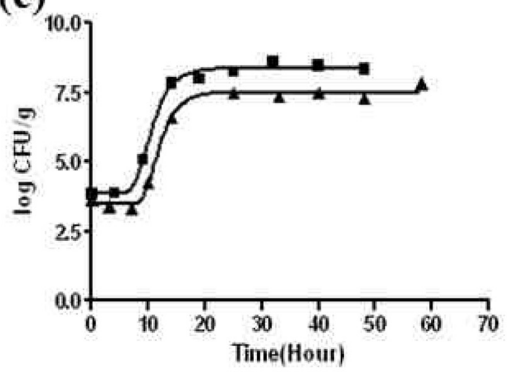

(e)

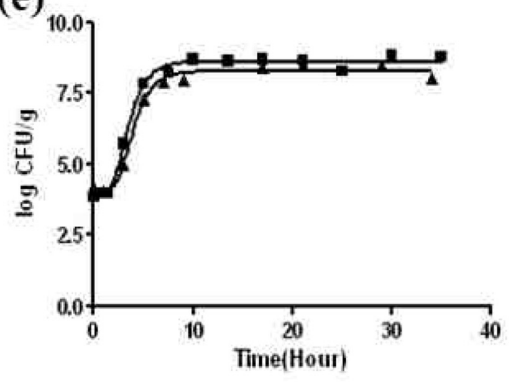

(b)

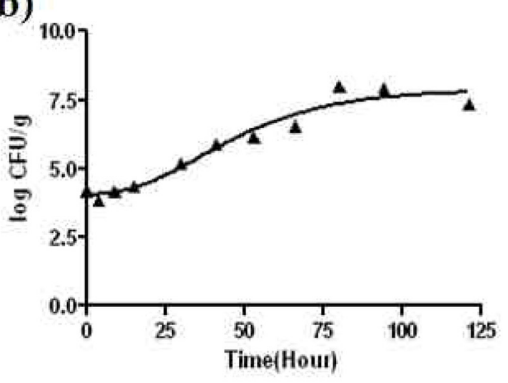

(d)

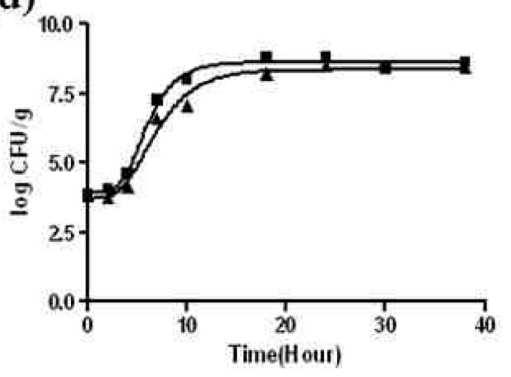

(f)

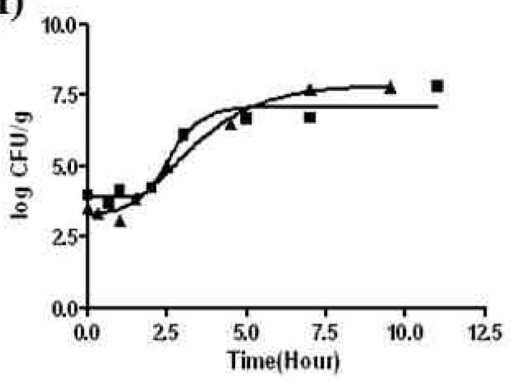

Fig. 1. Primary growth models of $C$. perfringens in anaerobically (b), $28^{\circ} \mathrm{C}(\mathrm{c}), 36^{\circ} \mathrm{C}(\mathrm{d}), 44^{\circ} \mathrm{C}(\mathrm{e})$, and $50^{\circ} \mathrm{C}(\mathrm{f})$, respectively. 
Table 2. Secondary model for lag time (LT), specific growth rate (SGR) and maximum population density (MPD) of $C$. perfringens in Jokbals by packaging method

\begin{tabular}{cclc}
\hline \hline Packaging & Parameter & \multicolumn{1}{c}{ Equation } & $\mathrm{R}^{2}$ \\
\hline \multirow{3}{*}{ anaerobically } & $\mathrm{LT}$ & ${ }^{1} \mathrm{Y}=6.72+(-516.5 / \mathrm{T})+\left(13398 / \mathrm{T}^{2}\right)$ & 0.991 \\
& $\mathrm{SGR}$ & ${ }^{2} \sqrt{\mathrm{Y}}=\{0.0352(\mathrm{~T}-7.912)\}$ & 0.994 \\
& $\mathrm{MPD}$ & ${ }^{3)} \mathrm{Y}=6.47+0.1353 \mathrm{~T}-0.0020 \mathrm{~T}^{2}$ & 0.964 \\
\hline \multirow{3}{*}{ aerobically } & $\mathrm{LT}$ & $\mathrm{Y}=9.23+(-764.9 / \mathrm{T})+\left(20304 / \mathrm{T}^{2}\right)$ & 0.988 \\
& $\mathrm{SGR}$ & $\sqrt{\mathrm{Y}}=\{0.0509(\mathrm{~T}-20.61)\}$ & 0.984 \\
& $\mathrm{MPD}$ & $\mathrm{Y}=2.63+0.3085 \mathrm{~T}-0.0041 \mathrm{~T}^{2}$ & 0.954 \\
\hline
\end{tabular}

${ }^{1)} \mathrm{Y}=\mathrm{a}+(\mathrm{b} / \mathrm{T})+\left(\mathrm{c} / \mathrm{T}^{2}\right)$

2) $\sqrt{\mathrm{Y}}=\left\{\mathrm{b}\left(\mathrm{T}-\mathrm{T}_{\min }\right)\right\}$

${ }^{3)} \mathrm{Y}=\mathrm{a}+\mathrm{bT}+\mathrm{cT}^{2}$

(a)

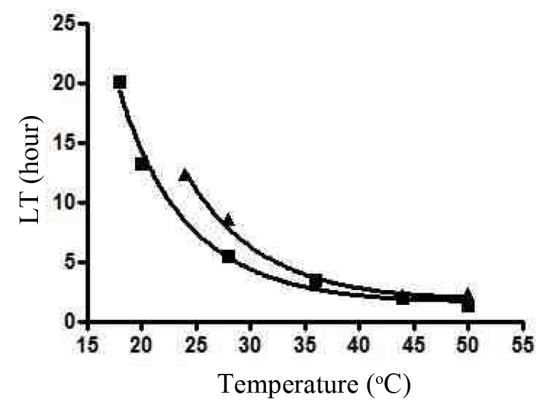

(c)

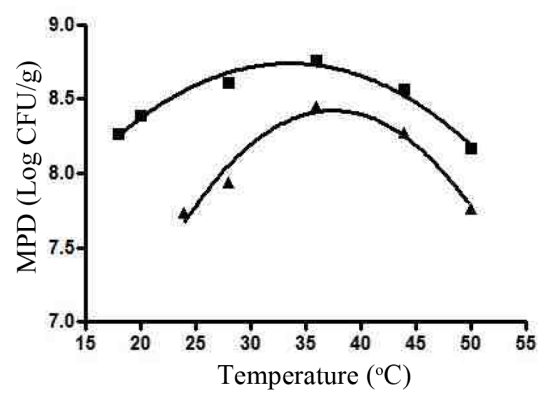

(b)

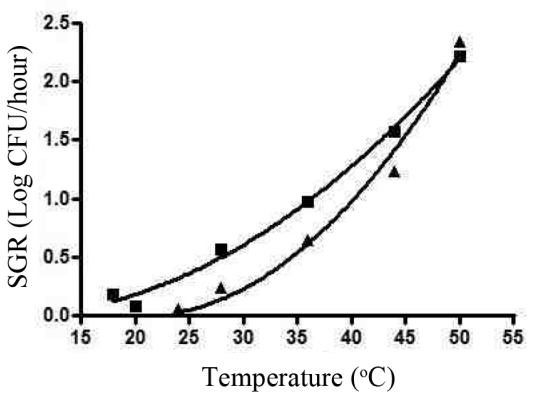

Fig. 2. Secondary models of the LT (a), SGR (b), and MPD (c) of C. perfringnes in Jokbals as a function of temperature anaerobically packaging, $\Delta$; aerobically packaging.

anaerobically packed Jokbal was faster than that of aerobically packed Jokbal at all tested temperatures. Significant differences in MPD values were also observed between anaerobically and aerobically packed Jokbals at $28^{\circ} \mathrm{C}$ and $50^{\circ} \mathrm{C}(p<0.05)$. These results indicate that the packaging method influences the growth kinetics of $C$. perfringens in Jokbals.

Optimal growth conditions for $C$. perfringens have been reported in previous works (Labbe and Juneja, 2001; McClane, 1997). C. perfringens growth has been shown at temperatures as high as $50^{\circ} \mathrm{C}$, while slowly arresting near $6^{\circ} \mathrm{C}$ and below (McClane, 1997). Labbe and Juneja (2001) also reported $43-45^{\circ} \mathrm{C}$ as an optimal growth temperature range with less than $10 \mathrm{~min}$ generation time. In addition, C. perfringens is capable of growth at relatively low water activities $\left(\mathrm{A}_{\mathrm{w}}\right)$ in the range of $0.93-0.97$ (Bartsch and Walker, 1982). Optimum $\mathrm{pH}$ for growth is between $\mathrm{pH} 6.0$ and 7.0 and most strains are inhibited by $5-6.5 \%$ salt, but the organism has been observed to grow at up to $8 \% \mathrm{NaCl}$ in foods (Johnson, 1990). The water activity of RTE Jokbal used in this study was 0.96 , which was optimum level for growth of $C$. perfringens. However, the $\mathrm{pH}$ of RTE Jokbal was 8.25 , which seems to be a limiting factor for the growth of $C$. perfringens at temperatures below $18^{\circ} \mathrm{C}$ in the present study. This outcome differs from the results of a previous study (McClane, 1997). Additionally, packaging method might influence the behavior of $C$. perfringens in this study. Modification of the atmosphere surrounding the food may provide a 'hurdle' that helps restrict the growth of an aerobic microorganism. Modified 
packaging methods resulted in more effective and safer storage regimes and longer shelf-life for meat products (Phillips, 2003). From the USDA-FSIS survey, 78\% of consumers store opened packages of deli meats for less than a week and around $10 \%$ of consumers keep them for $1-3 \mathrm{wk}$ in their refrigerators (USDA-FSIS, 2003). Juneja et al. (1994) evaluated the potential for growth of C. perfringens in aerobically and anaerobically packed cooked ground turkey. At $28^{\circ} \mathrm{C}$, The organism grew up to $7 \operatorname{logs}$ anaerobically within $9 \mathrm{~h}$ and $24 \mathrm{~h}$ under aerobic conditions. In addition the lag time of anaerobically and aerobically packed ground turkey at $28^{\circ} \mathrm{C}$ was $2.57 \mathrm{~h}$ and $7.47 \mathrm{~h}$, respectively. The result of the present study is similar to their work. In this study, C. perfringens grew up to $8 \operatorname{logs}$ within $9 \mathrm{~h}$ anaerobically and $12.5 \mathrm{~h}$ aerobically at $36^{\circ} \mathrm{C}$. Thus anaerobic microorganisms such as $C$. perfringens have potential hazards under anaerobic packaging method. Especially, temperature abuse of precooked, anaerobic packaging products, such as Jokbal, for rela-

(a)

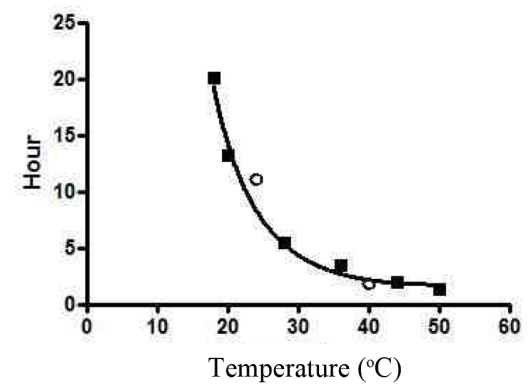

(c)

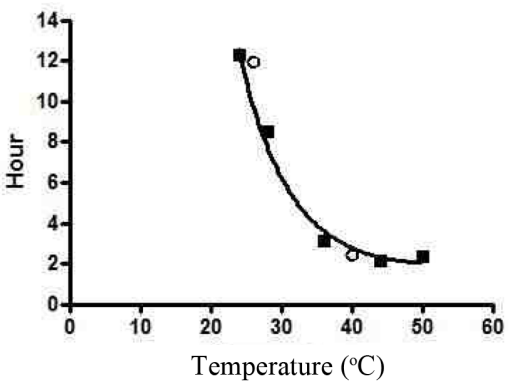

(e)

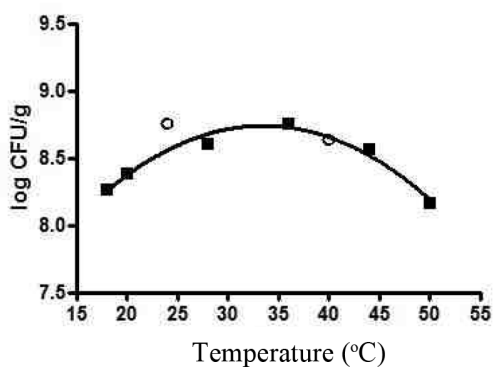

tively long periods may lead to high and dangerous numbers of $C$. perfringens at retail markets. According to the results of the survey study (Part et al., 2013), 80.1\% of consumers responded that they bought Jokbal that was stored at room temperature.

\section{Secondary growth model of $C$. perfringens in Jok- bal and model performance}

Secondary models were also developed to describe the effect of storage temperature and packaging method on the primary model parameters, including LT and SGR, and MPD (Table 2). Fig. 2 shows a comparison of the secondary growth models of $C$. perfringens in anaerobic and aerobically packed RTE Jokbal. The Davey model for LT (Fig. 2(a)), square root model for SGR (Fig. 2(b)), and polynomial model for MPD (Fig. 2(c)) had a high goodness-of fit for the parameters of the primary growth model of $C$. perfingens in RTE Jokbal.

Secondary models were also evaluated for their ability

(b)

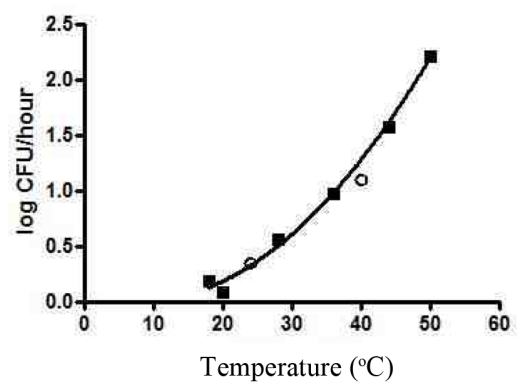

(d)

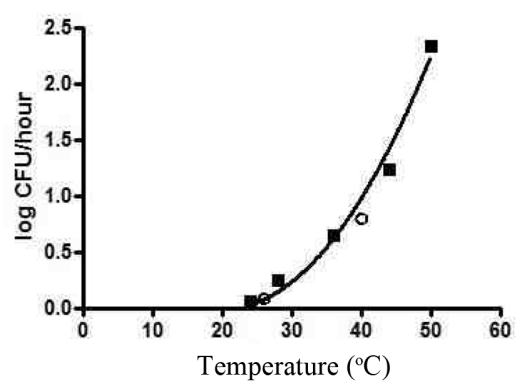

(I)

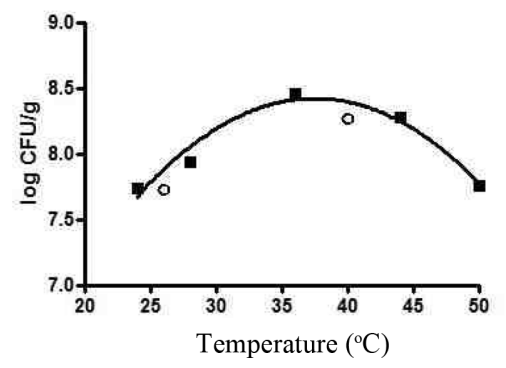

Fig. 3. Secondary LT, SGR, and MPD models for $C$. perfringens in Jokbals as a function of packaging and temperature. Anaerobically: (a) LT, (c) SGR, (e) MPD, Aerobically: (b) LT, (d) SGR, (f) MPD. $\square$;Dependent data, $\bigcirc$; Independent data. 
Table 3. Model Performance of secondary models of C. perfringens in Jokbals

\begin{tabular}{ccccccc}
\hline \hline \multirow{2}{*}{ Packaging } & \multirow{2}{*}{ Model } & \multicolumn{2}{c}{ Goodness-of-fit } & \multicolumn{2}{c}{ Prediction bias } & \multicolumn{2}{c}{ Prediction accuracy } \\
\cline { 2 - 7 } & & ${ }^{10} \mathrm{R}^{2}$ & ${ }^{2} B_{f}$ & ${ }^{3)} \mathrm{MRE}$ & ${ }^{4)} A_{f}$ & ${ }^{5} \mathrm{MARE}$ \\
\hline \multirow{3}{*}{ Anaerobic } & LT & 0.991 & 1.00 & 0.02 & 1.13 & 0.12 \\
& SGR & 0.994 & 1.16 & 4.99 & 1.28 & 0.33 \\
& MPD & 0.964 & 1.00 & 0.01 & 1.01 & 0.00 \\
\hline \multirow{3}{*}{ Aerobic } & LT & 0.988 & 1.00 & 0.01 & 1.15 & 0.14 \\
& SGR & 0.984 & 0.95 & 0.12 & 1.01 & 0.20 \\
& MPD & 0.954 & 1.00 & 0.00 & & 0.01 \\
\hline
\end{tabular}

${ }^{1} \mathrm{R}^{2}$ : Coefficient of determination

${ }^{2)} B_{f}$ : Bias factor

${ }^{3)} \mathrm{MRE}$ : Median relative error

${ }^{4)} A_{f}$ : Accuracy factor

${ }^{5)}$ MARE : Mean absolute relative error

to interpolate within the response surface using independent temperature data not used in model development (Fig. 3). These data were collected with the same experimental design of the present study at 24 and $40^{\circ} \mathrm{C}$ for anaerobically packaged Jokbal and 26 and $40^{\circ} \mathrm{C}$ for aerobically packaged Jokbal. As shown in Fig. 3, both dependent and independent (interpolation) data fitted well to secondary models. Thus, model performance of secondary models for LT, SGR and MPD of C. perfringens in Jokbal was validated for interpolation with bias factor $\left(B_{f}\right)$ and accuracy factor $\left(A_{f}\right)$ (Table 3$)$. The acceptable ranges of $B_{f}$ are 0.7-1.15 and a range of 0.9-1.05 is considered to be good (Ross, 1996). The LT, SGR, and MPD models for anaerobically packed Jokbal including interpolation data had the $B_{f}$ values of $1.00,1.16$, and 1.00 , respectively. These results indicated that the developed model for anaerobically packed Jokbals predicted a SGR that was $16 \%$ lower than the actual experimental values. The LT, SGR, and MPD models for aerobically packed Jokbal including interpolation data had the $B_{f}$ values of $1.00,0.95$, and 1.00, respectively. This result indicates that the developed model for aerobically packed Jokbals predicted a SGR that was $5 \%$ higher than the actual experimental values.

In conclusion, the growth of $C$. perfringens in anaerobically packed Jokbal was more rapid than that in aerobically packed Jokbal. Packaging method also influences the minimum growth temperatures of $C$. perfringens in RTE Jokbal. Thus anaerobic microorganisms, such as $C$. perfringens, have a potential hazard in RTE anaerobically packed Jokbal at retail markets. Temperature abuses between 18 to $50^{\circ} \mathrm{C}$ may especially lead to dangerous numbers of C. perfringens in Jokbal at retail markets. This presents a significant risk to public health. In addition, the developed secondary LT, SGR, and MPD models were able to reliably predict the growth of $C$. perfringens in aerobically and anaerobically packed Jokbal. This may also pro- vide useful information for microbial risk assessment of C. perfingens in RTE foods containing meat, such as packaged meals called "dosirak," which are sold in convenience stores.

\section{Acknowledgements}

This research was supported by the Korea Food Drug Administration Research Grant (11162-044).

\section{References}

1. Abou-Zeid, K. A.,Oscar, T. P., Schwarz, J. G., Hashem, F. M., Whiting, R. C., and Yoon, K. S. (2009) Development and validation of a predictive model for Listeria monocytogenes Scott $\mathrm{A}$ as a function of temperature, $\mathrm{pH}$, and commercial mixture of potassium lactate and sodium diacetate. J. Microbiol. Biotechnol. 19, 718-726.

2. Bartsch, A. and Walker, H. (1982) Effect of temperature, solute and $\mathrm{pH}$ on the tolerance of Clostridium perfringens to reduced water activities. J. Food Sci. 47, 1754-1755.

3. Bryan, F. L. (1988) Risks of practices, procedures and processes that lead to outbreaks of foodborne diseases. J. Food Prot. 51, 663-673.

4. Brynestad, S. and Granum, P. E. (2002) Clostridium perfringens and foodborne infections. Int. J. Food Microbiol. 74, 195-202.

5. Daughtry, B. J., Davey, K. R., and King, K. D. (1997) Temperature dependence of growth kinetics of food bacteria. Food Microbiol. 14, 21-30.

6. Delignette-Muller, M. L., Rosso, L., and Flandrois, J. P. (1995) Accuracy of microbial growth predictions with square root and polynomial models. Int. J. Food Microbiol. 27, 139-146.

7. Gibson, A. M., Bratchell, N., and Roberts, T. A. (1987) The effect of sodium chloride and temperature on the rate and extent of growth of Clostridium botulinum type A in pasteurized pork slurry. J. App. Bacteriol. 62, 479-490.

8. Golden, N. J., Crouch, E. A., Latimer, H., Kadry, A. R., and Kause, J. (2009) Risk assessment for Clostridium perfringens 
in ready-to-eat and partially cooked meat and poultry products. J. Food Prot. 72, 1376-1384.

9. Hatheway, C. L. (1990) Toxigenic clostridia. Clin Microbiol. Rev. 3, 66-98.

10. Juneja, V., Call, J., Marmer, B., and Miller, A. (1994) The effect of temperature abuse on Clostridium perfringens in cooked turkey stored under air and vacuum. Food Microbiol. 11, 187-193.

11. Juneja, V. K., Marks, H., and Thippareddi, H. H. (2008) Predictive model for growth of Clostridium perfringens during cooling of cooked uncured beef. Food Microbiol. 25, 42-55.

12. Juneja, V. K., Marks, H., and Thippareddi, H. H. (2010) Predictive model for growth of Clostridium perfringens during cooling of cooked ground pork. Innovative Food Sci. Emerging Technol. 11, 146-154.

13. Johnson, E. A. (1990) Clostridium perfringens food poisoning. In foodborne Dieases. D. O. Cliver (ed.) Academic Prss Inc., California.

14. Johnson, S. and Gerding, D. (1997) Enterotoxemic infection. In: The Clostridia: Molecular Biology and Pathogenesis. J. I. Rood, B. A. McClane, J. G. Songer and R. W. Titball (eds.) Academic Press, London, pp. 117-140.

15. Labbe, R. G. and Harmon, S. M. (1992) Clostridium perfringens, In C. Vanderzant and D. F. Splittstoesser (ed.), Compendium of Methods for the Microbiological Examination of Foods, 3rd Edition. Amer. Publ. Health Assoc., Washington, D.C. pp. 623-635.

16. Labbe, R. G. and Juneja, V. K. (2001) Clostridium perfringens. In Foodborne infections and intoxications. H. Riemann and F. L. Bryan (eds.) Academic press, NY.

17. Le Marc, Y., Plowman, J., Aldus, C. F., Munoz-Cuevas, M.,
Baranyi, J., and Peck, M. W. (2008) Modelling the growth of Clostridium perfringens during the cooling of bulk meat. Int. J. Food Microbiol. 128, 41-50.

18. McClane, B. A. (1997) Clostridium perfringens. In Food microbiology: Fundamentals and frontiers. M. P. Doyle, L. R. Bueuchat and T. J. Montville (eds.) ASM Press, Washington, DC. pp. 305-326.

19. McMeekin, T. A., Olley, J., and Ross, T. (1993) Predictive microbiology: Theory and application. John Wiley \& Sons Ltd., Taunton, UK. p. 340.

20. MFDS (2013) Statistics of Foodborne illness. Available from: http://www.mfds.go.kr/e-stat/index.do?nMenuCode=20

21. Park, H. J., Min, K. J., Park, N. Y., Cho, J. I., Lee, S. H., Hwang, I. G., Heo, J. J., and Yoon, K. S. (2013) Estimation on the consumption patterns of potentially hazardous foods with high consumer risk perception. Korean J. Food Sci. Technol. 45, 59-69.

22. Phillips, C. A. (2003) Review: modified atmosphere packaging and its effects on the microbiological quality and safety of produce. Int. J. Food Sci. Tech. 31, 463-479.

23. Ratkowsky, D. A., Olley, J., McMeekin, T. A., and Ball, A. (1982) Relationship between temperature and growth rate of bacterial cultures. J. Bacteriol. 149, 1-5.

24. Ross, T. (1996) Indices for performance evaluation of predictive models in food microbiology. J. Appl Bacteriol. 81, 501508.

25. USDA-FSIS (2003) Consumer attitudes and behaviors regarding ready-to-eat foods. Available from: http://www.fsis.usda. gov/OPPDE/rdad/FRPubs/02-041N/conley_lm.htm. Accessed Feb., 10, 2013.

(Received 2013.9.10/Revised 2014.1.22/Accepted 2014.1.22) 